\title{
A Novel Algorithm for AODV to Enhance the Efficiency and Performance of the MANET
}

\author{
${ }^{1}$ Deepika J, ${ }^{2}$ Dr. Rangaiah L \\ ${ }^{1}$ Research scholar, Department of ECE, RajaRajeswari College of Engineering, Bangalore, under VTU, Belgavi, India. \\ ${ }^{2}$ Professor \& Head,Department of ECE, RajaRajeswari College of Engineering, Bangalore,560074, India. \\ Article History: Received:11 January 2021; Accepted: 27 February 2021; Published online: 5 April 2021 \\ Abstract:Network security and performance are significant topics discussed in academic and Industrial research. Every \\ application depends on the performance and efficiency of the network. Routing protocols and mechanisms play an \\ essential role. AODV routing protocol is considered standard in many aspects, primarily due to the increasing efficiency \\ requirements in various applications. There are different approaches in the literature trying to improve the performance. \\ Some studies considered multiple parameters such as density and mobility of the data nodes. This research study \\ evaluates the two critical parameters that contribute to the overall efficiency and performance of the network - \\ throughput and route resilience. A new version of enhanced AODV is proposed in this paper that uses an iterative \\ approach to check for each parameter and adjust the values accordingly until the required threshold level of efficiency is \\ reached. The solution is explained in different categories pertaining to each network parameter. The proposed algorithm \\ is simulated on a network tool, and the results show that the enhanced version of the routing protocol is promising and \\ can be further extended to introduce new modes for higher efficiency and lower power consumption.
}

Index Terms: Network performance, AODV, Throughput, Route Resilience, new algorithm, Routing protocol, network simulation, OMNeT++ tool.

\section{Introduction}

Networking is a vast field with many technologies that are evolving almost every day. Networks are now able to transmit a large amount of information at high speeds over the internet and wireless medium. Wireless ad hoc networks are a particular case of wireless networks. The primary characteristic of this network is that it does not rely on any specific architecture or infrastructure, as in the case of wired networks. Every node is capable of sending and receiving the data by routing. With this capability of the nodes, there is no specific order in which the nodes are assigned sender and receiver. Routing algorithms are used to enable the networks to transmit information, and the nodes are automatically assigned based on the routing mechanism. The significant advantage of the Ad-Hoc network is that the complexity of the infrastructure required to set up the network is minimized. The nodes are now capable of joining and leaving the network at any point without any hurdle [3].

Wireless Ad Hoc networks are capable of configuring without any external infrastructure or connections to the dynamic networks when the nodes are mobile. Mobile Ad Hoc networks are capable of multicast routing along with unicast.

\section{Related work}

There are two popular categories of routing protocols used in MANETs. These protocols are classified based on the mechanism used. Proactive protocols are specific in their mechanism and use routing tables. The proactive mechanism requires the information to be available in the routing table with the details of every route and data packet. The dynamic nature of MANET requires changes to the routing tables and route information. Destination Sequenced Distance Vector (DSDV) Routing Protocol is an example of a proactive routing protocol. The second category is a reactive protocol in which the routing table is not maintained with the information required; instead, the requests from the data packet are considered as the initiative to discover the outside and transmit the data. The route discovery process is initiated as soon as the requests are initialized. There are many advantages of this mechanism, especially with resource utilization, because there are no specific routing protocols are tables maintained at all times. Ad-hoc On-demand Distance Vector (AODV) routing is a popular routing protocol used in MANET [5].

Literature work in the field of AODV and routing protocols is extensive. There are numerous protocols and parameters used to measure efficiency. There are specific metrics used by the researchers to analyze the performance and efficiency of each routing protocol. There are different applications for these protocols, and it is a challenge to check the comparison and proposed appropriate method. Not all the comparison metrics use the same application, and it is a challenge for the researchers to analyze all the applications that use a routing protocol. Some of the common metrics used are throughput, route discovery, packet delivery [6].

Performance of the wireless networks is of primary importance for every application. AODV research is extensive because of the advantages it provides, and many research studies have concentrated on improving the efficiency using control schemes and adjustments to the parameters. The routing condition is described before the data packet is initialized in a new control scheme proposed in the literature. A congestion control mechanism is designed to manage the condition by analyzing the entry of the values in the routing table. The results were simulated, and it was found that the understanding of the routing table values is critical because the details of the routing and the possibility of packet drop can be analyzed with this information. The knowledge of routing tables is not only crucial for improving the condition control but can also improve the throughput and minimize the Drop rate [2].

A research study was designed and implemented in which the range of the transmission and its connection with the density of the node was analyzed. The transmission range influences the density of the node, and it was found that the 
increase in density resulted in a decrease of the delay in the new enhanced method when compared to AODV. This research study and results are more appropriate to an application with more node density. The density of the nodes and the region we are not considered in the current research study, but the existing studies show that the parameters such as packet delivery and network throughput can cause significant influence on the efficiency of the system [1]. AODV routing protocol is not restricted to Ad Hoc networks and wireless sensor networks but can also be easily extended to IoT applications.

A common approach used by the researchers in this field is the comparison of AODV routing protocol with other protocols for a specific application. Packet delivery rate and drop rate are considered to be effective measures of comparing the efficiency. Dynamic routing protocols have been proposed in the literature for addressing specific problems such as the mobility of the nodes. The results of the research studies have also been compared with other protocols such as OLSR, and it was found that AODV has better performance [7]. RREP and RREQ are the packets sent in the routing protocol, which increases the traffic when there are too many data packets in the network, and it is overloaded. The functionality and performance of the system deteriorate. The research literature provides proof on using various metrics, but the common feature found in many studies relates to the two critical parameters - route resilience and throughput. This research study proposes an enhanced AODV design with throughput, route resilience, and power consumption parameters.

\section{Design}

The design of the enhanced network is divided into two parameters - Throughput and route resilience. Message rate, communication rate, and data rate metrics are used to measure these parameters. The first step is to check if the routes are available for data transmission. Data values in the round table represent the different nodes and the path to be taken by the data packets. The information from the routing table may or may not be available depending on the design of the network [8]. If the route is not available in the table, the process of discovery is initiated in which the possible mathematical calculations for discovering the new route are initialized. If the routes are available in the table, the next step is to check for the communication range.

A small set of test packets are sent through the communication channel, and the throughput is recorded. A preferred value of throughput is compared with the threshold value. The necessary condition is to have the throughput greater than the threshold value so that the communication and the data packets last for the entire session, as shown in figure 1. If the throughput is less than the threshold, then the communication range is increased until the value increases beyond the threshold.

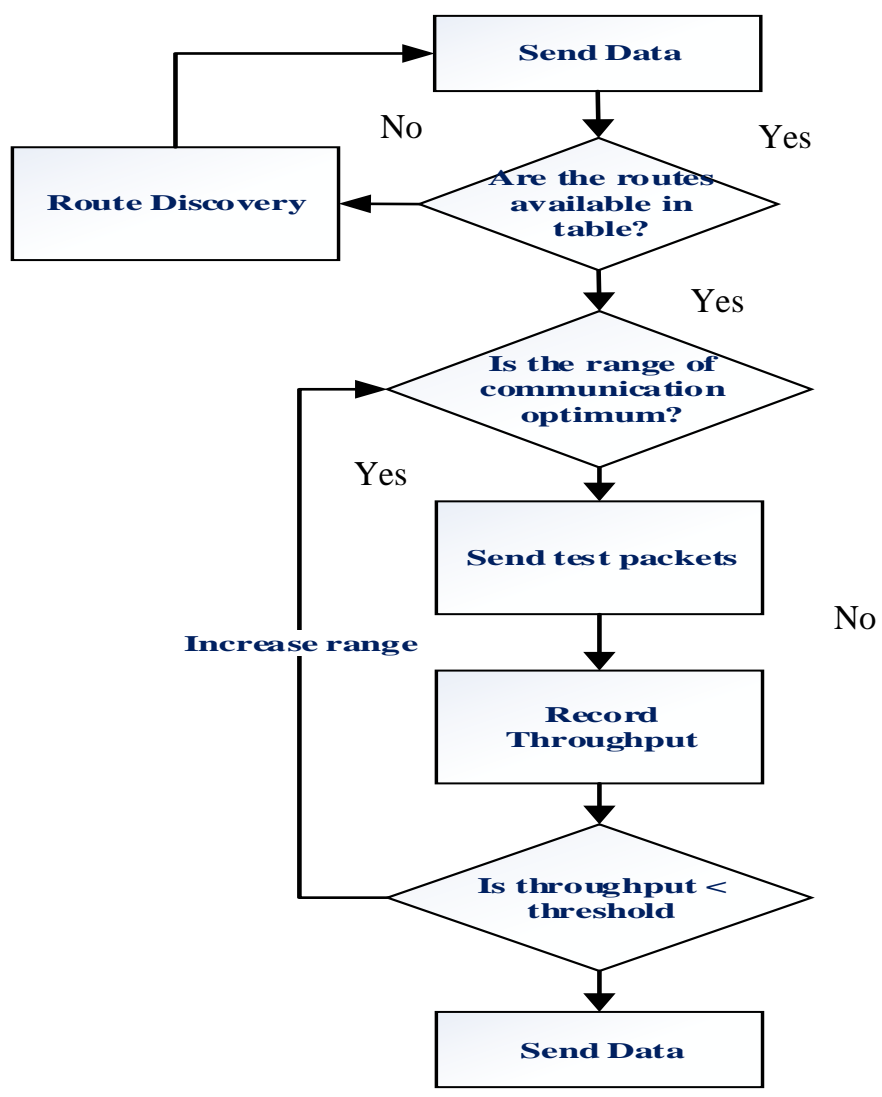

Fig.1. Communication Range optimization using new algorithm 
A new enhanced algorithm is designed in which the start time and acknowledge the time of the data packet are recorded. This data is compared with the standards for data rate in which noiseless channels are used for improving efficiency. The data rate is calculated for the initial stages when the required starting time is determined. The throughput is calculated after determining the data rate and compared with the threshold level, as shown in figure 2 . The signal levels are increased for enhanced communication only after checking the data parameters, the highlight of the improved algorithm. The route Discovery process should not be advertised to all the nodes, especially when there are specific adjustments to be made with the communication range and message rate [9]. If the route Discovery process is advertised, the control packet data is increased, and the network is flooded with additional data packets. The route discovery should always result in updating the values in the routing table

In a slightly different enhancement of the existing algorithm, the first step is to check if the roots are available in the routing table and then proceed with the reachability of the node. If the node is not reachable, an alternative route is checked, as shown in figure 3.

If no alternative is available, then the route discovery process is initiated. This enhanced version of the flowchart provides an additional requirement of checking if an alternative route is available, which makes the algorithm efficient because the route discovery process is delayed unless absolutely necessary [10]

Fig.2. Enhanced data rate with a new algorithm

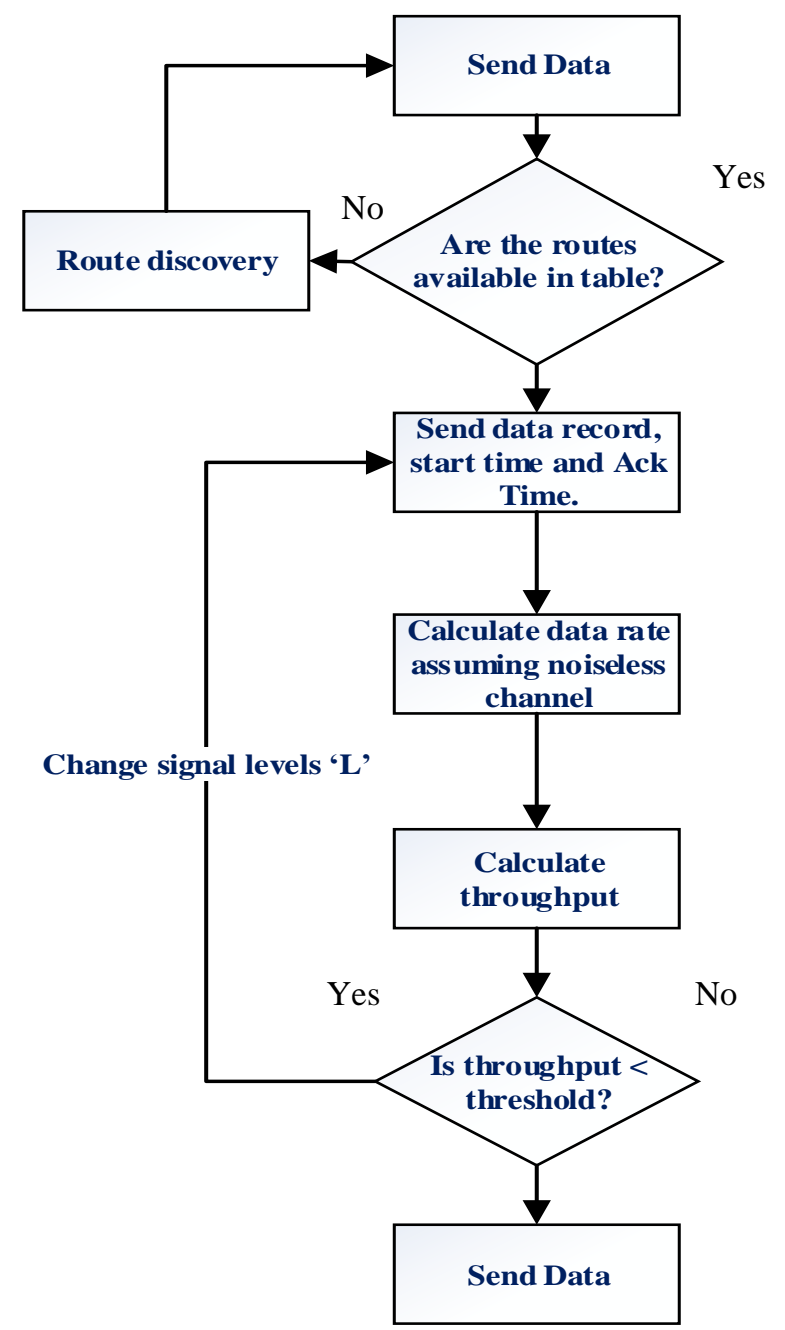




\section{Results}

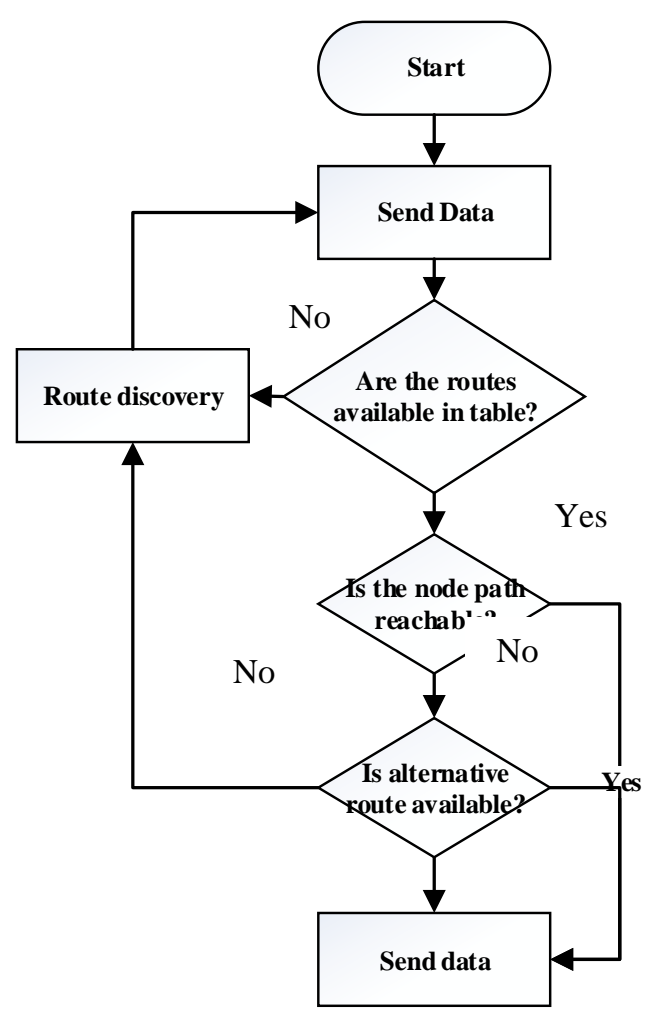

Fig.3. Route Resilience using a new algorithm

The proposed routing protocol based on AODV is simulated on the OMNeT++ tool. Various libraries are available on the tool, and the $\mathrm{C}++$ framework is used for designing and coding. This software tool is available free of cost for non-commercial purposes and academic projects. The total number of packets sent in the simulation are 1662.The results are discussed as follows.

Default Network - Number of packets sent - 1662, Number of packets received - 326

Enhanced Network - Number of packets sent - 1662, Number of packets received - 1127

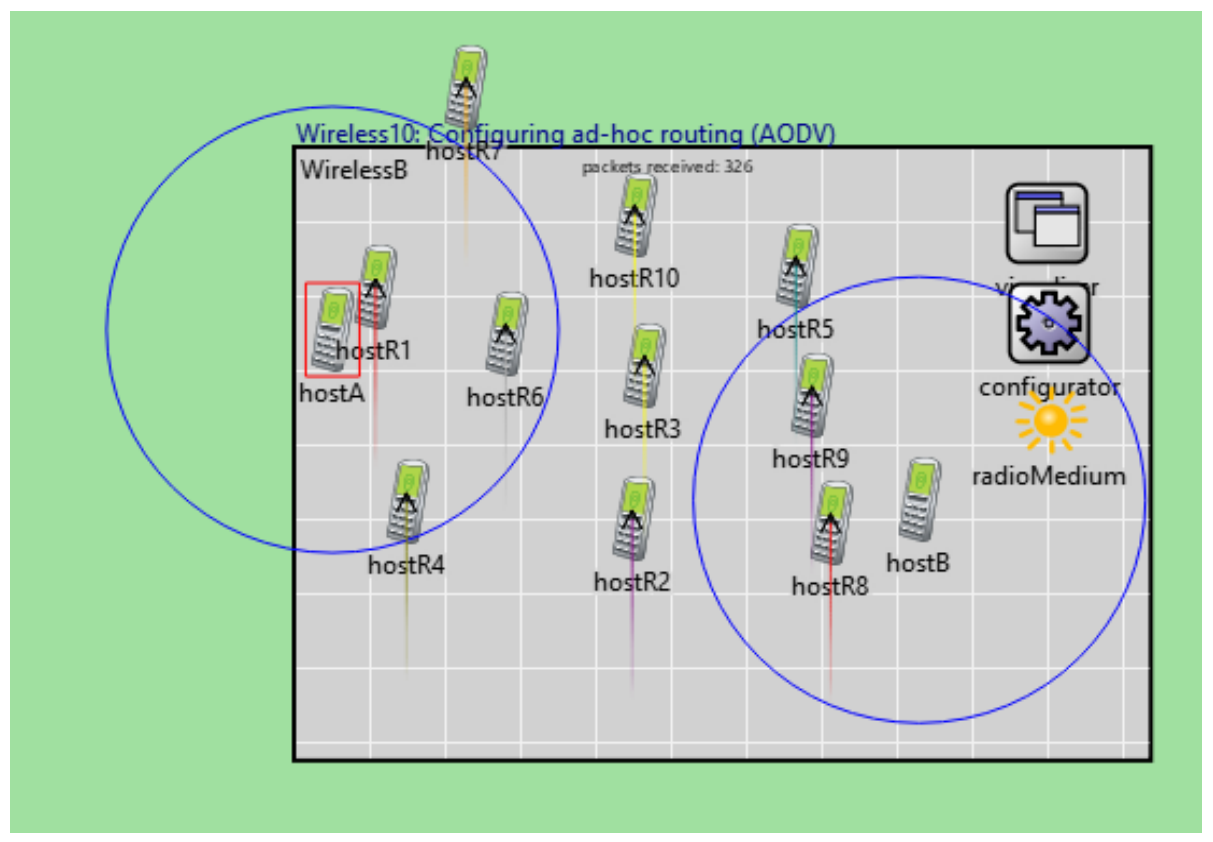

Fig.4. 326 packets received in default network 


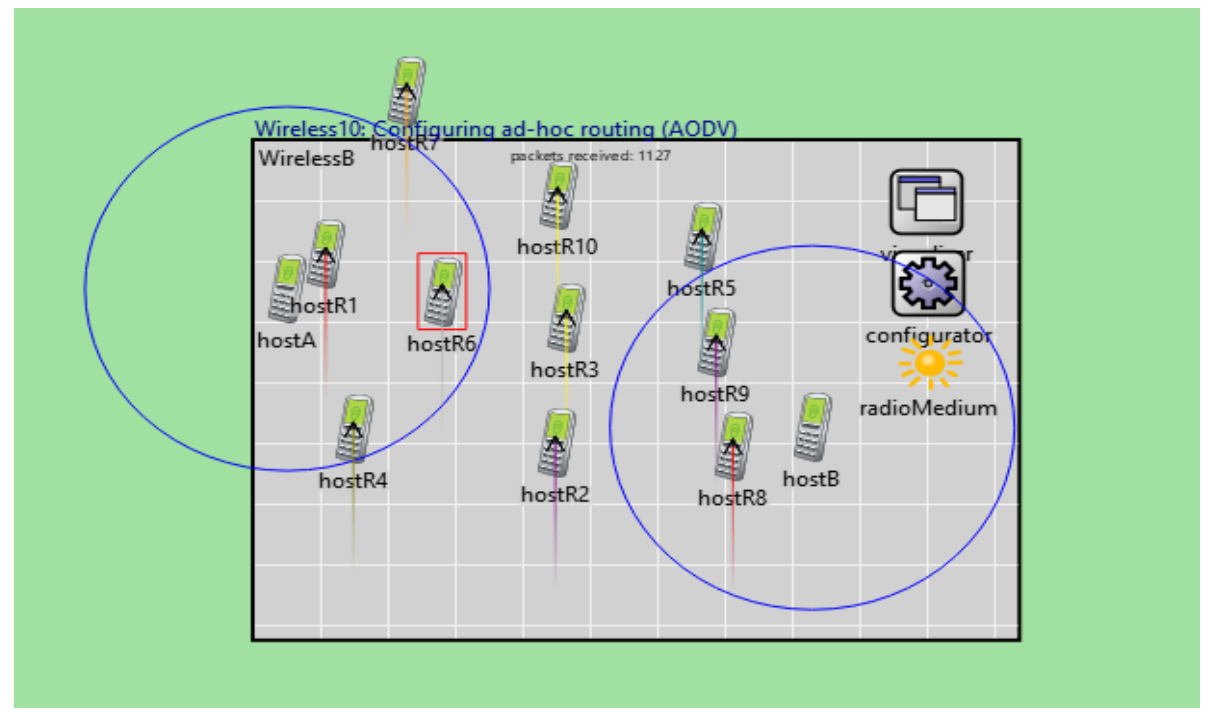

Fig.5. 1127 packets received in enhanced network

Throughput is checked using the default Network and enhanced network simulation. The network diagrams of both default and enhanced networks are displayed with 326 packets and 1127 packets received, respectively. The number of intermediate nodes is changed for the enhanced network, and the comparison is given for different intermediate nodes [9]. Intermediate nodes were increased from 25 to 100. It is clear that the packets received decreases with the increase in the intermediate nodes, as shown in figure 7.

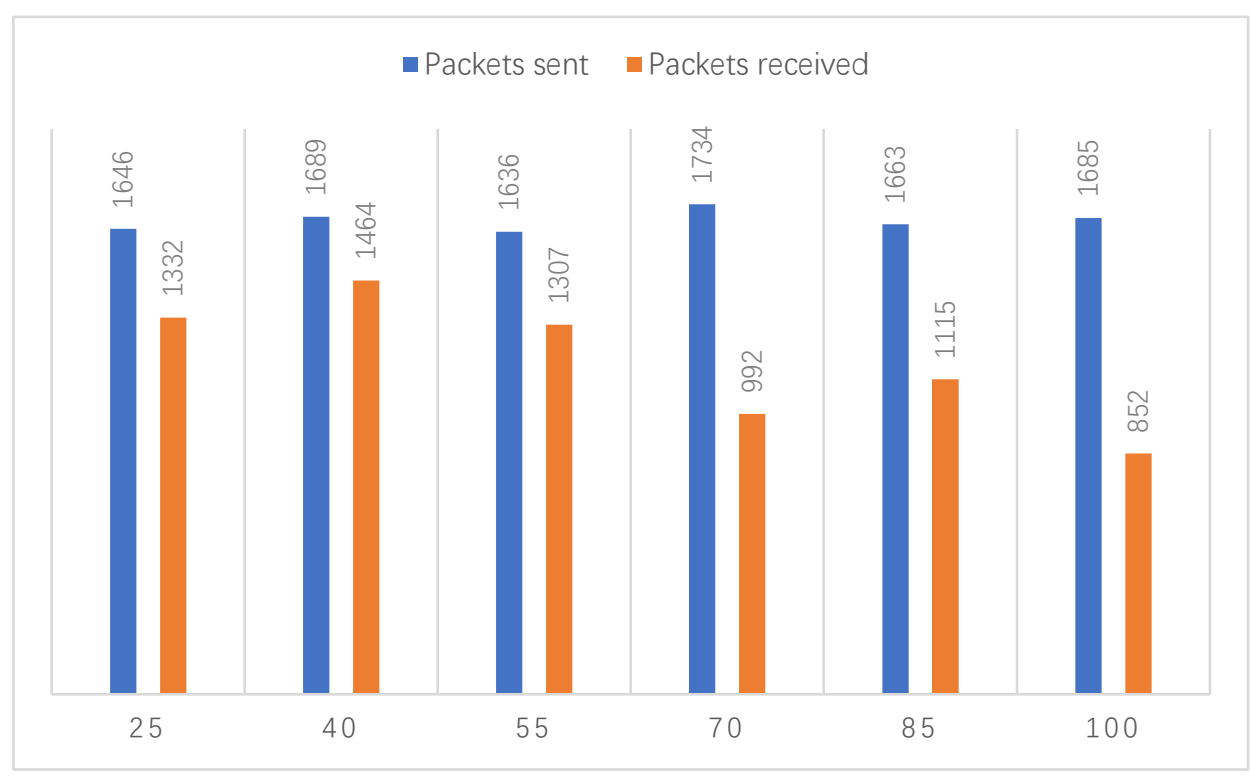

Fig.7.Throughput comparison for different intermediate nodes

The communication range was also checked using simulation, and the message size of 1000B was used, and the data packets sent and received are compared. The throughput values were found to be increasing for a higher communication range measured from $<1 \mathrm{~m}$ to $500 \mathrm{~m}$. 


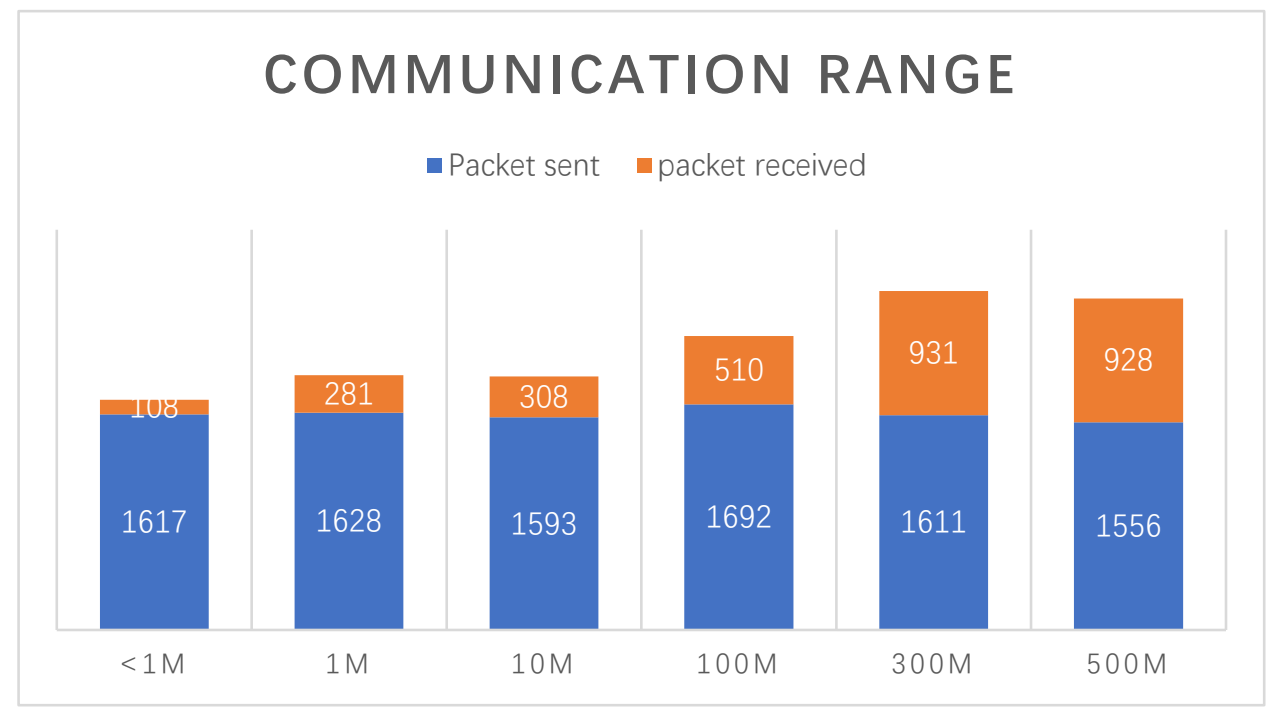

Figure 8. Communication range and packets received.

The graph that compares the packet sent and received for different data rates ranging from 1Mbps to $10 \mathrm{Mbps}$ is plotted. The chart clearly shows that the data packets received are increasing steadily as the data rates are increased (figure 8). The network is iterative in nature and found to perform efficiently. AODV is efficient only for smaller networks but the enhanced network is found to have higher efficiency for large networks with many nodes.

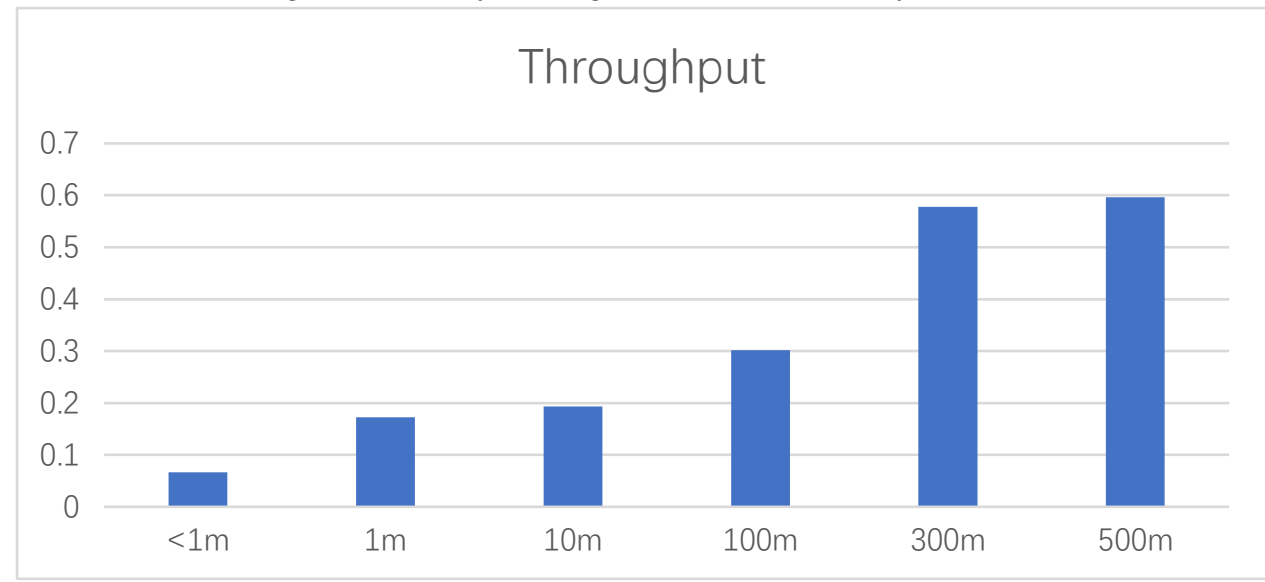

Figure 9. Throughput comparison for varying communication range

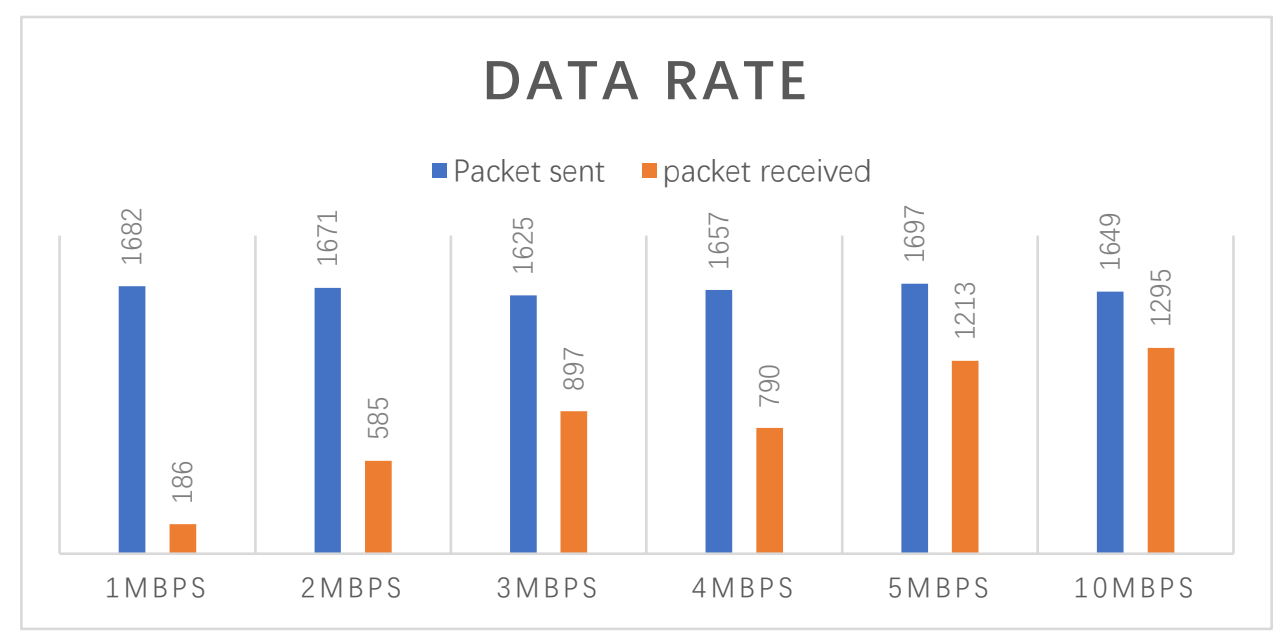

Figure 10. Data rate and packet received 


\section{Route Resilience}

Route resilience is the concept used to enhance the efficiency by increasing the throughput. The approach used for this is to check for different routes. The proposed enhanced algorithm checks for alternative routes and the simulation shows that this approach is efficient in improving the throughput. The enhanced algorithm has resulted in a throughput value of 0.43719 compared to default network with 0.19614 .

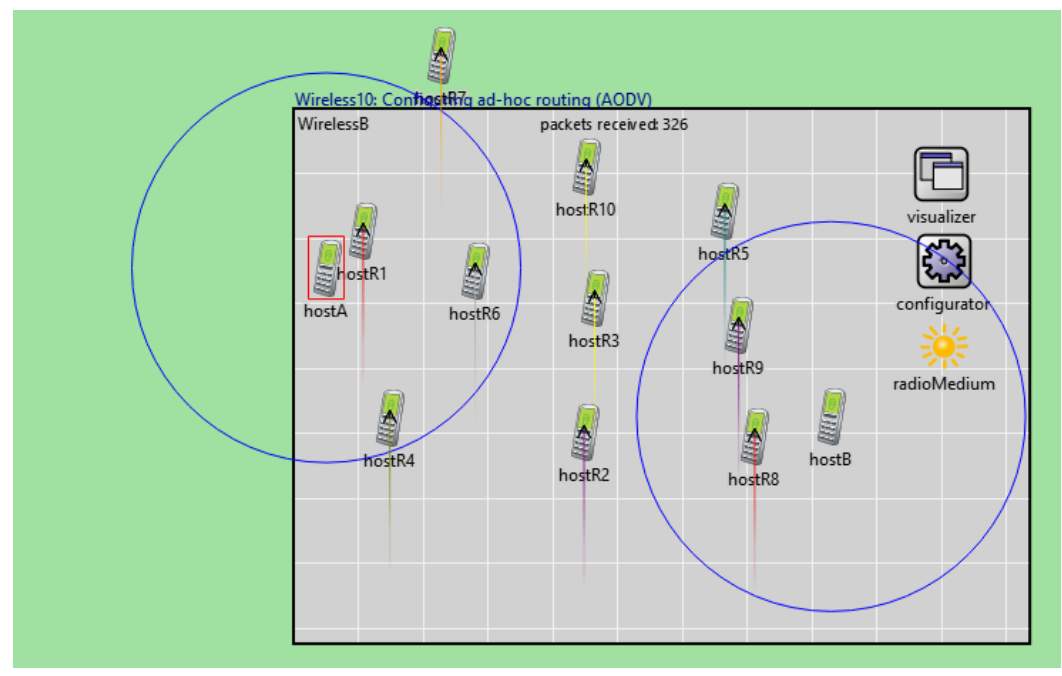

Figure 11. 326 Packets in the default network for route resilience

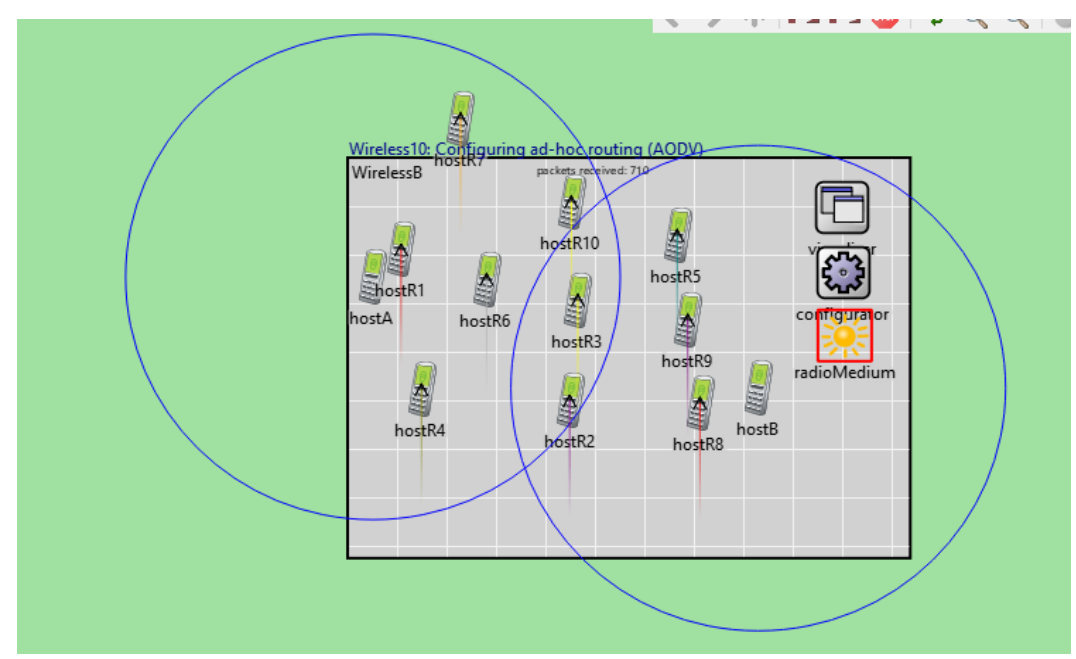

Figure 12. Enhanced Network - 710 Packets Received for route resilience

Default Network - Number of packets sent - 1662, Number of packets received -326 .

Enhanced Network - Number of packets sent -1624 , Number of packets received -710 .

\section{Conclusion}

This research paper proposes a new enhancement to the standard network and simulates the results by varying various parameters. This research study simulated the existing AODV routing protocol with specific parameters considered and enhanced the method using an iterative approach by adjusting various parameters to increase the efficiency and throughput. Throughput and route resilience are analyzed in this study. Route resilience parameter has shown significant improvement in throughput. Power consumption parameter is considered the future scope of the study. It is possible to achieve low power operations on MANETs and wireless sensor networks. 


\section{References}

1. Bamhdi, A.M., 2020. Efficient dynamic-power AODV routing protocol based on node density. Computer Standards \& Interfaces, 70, p.103406.

2. Mai, Y., Rodriguez, F.M. and Wang, N., 2018, January. CC-ADOV: An effective multiple paths congestion control AODV. In 2018 IEEE 8th Annual Computing and Communication Workshop and Conference (CCWC) (pp. 1000-1004). IEEE.

3. Anamalamudi, S., Sangi, A.R., Alkatheiri, M. and Ahmed, A.M., 2018. AODV routing protocol for Cognitive radio access based Internet of Things (IoT). Future Generation Computer Systems, 83, pp.228-238.

4. Hassan, M.H., Mostafa, S.A., Mohammed, M.A., Ibrahim, D.A., Khalaf, B.A. and Al-Khaleefa, A.S., 2019. Integrating African Buffalo optimization algorithm in AODV routing protocol for improving the QoS of MANET. Journal of Southwest Jiaotong University, 54(3).

5. Saini, T.K. and Sharma, S.C., 2020. Recent advancements, review analysis, and extensions of the AODV with the illustration of the applied concept. Ad Hoc Networks, 103, p.102148.

6. Zrelli, A., Khlaifi, H. and Ezzedine, T., 2019, September. Performance Evaluation of AODV and OAODV for Several WSN/IoT Applications. In 2019 International Conference on Software, Telecommunications and Computer Networks (SoftCOM) (pp. 1-6). IEEE.

7. Chouhan, A.S., Sharma, V., Singh, U. and Sharma, R., 2017, April. A modified AODV protocol to detect and prevent the wormhole using hybrid technique. In 2017 International conference of Electronics, Communication and Aerospace Technology (ICECA) (Vol. 2, pp. 621-625). IEEE.

8. Anand, M. and Sasikala, T., 2019. Efficient energy optimization in mobile ad hoc network (MANET) using better-quality AODV protocol. Cluster Computing, 22(5), pp.12681-12687.

9. Kumar, S., Dhull, K., Arora, P. and Luhach, A.K., 2019. Performance of Energy Conservation Models, Generic, Micaz and Micamotes, using AODV Routing Protocol on a Wireless Sensor Network. Scalable Computing: Practice and Experience, 20(4), pp.631-639.

10. Sarkar, D., Choudhury, S. and Majumder, A., 2018. Enhanced-Ant-AODV for optimal route selection in mobile ad-hoc network. Journal of King Saud University-Computer and Information Sciences.

\section{Authors' Profiles}

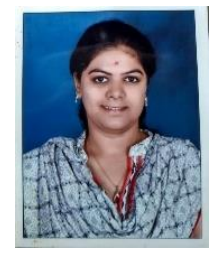

DeepikaJ working as an Assistant professor in the department of ECE of RajaRajeswariCollege of Engineering, Bangalore. She has completed her B. E and M.Tech in ACS college of Engineering, Bangalore. She is a Research scholar pursuing her doctorate degree in Engineering in RRCE Research center, Bangalore Under Visvesvaraya Technological University, Belgavi, India. Her area of research is on Mobile Ad-Hoc Network.

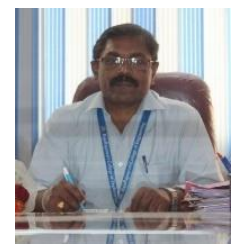

Dr. L Rangaiah holds Bachelor degree in Electronics and Communication Engineering from NBKR Institute of Science and Technology affiliated to S V University, M.Tech in Computer and Communication Engineering from Bharath University and Ph. D from University of Allahabad, Uttar Pradesh. He is having 23 years of experience in teaching and research and published 33 papers in National, International Conferences and International journals. Fellow Member of IETE and IE(I) and Member of MISTE, IEEE and MIAEng. His areas of interest are VLSI and Wireless Communications. Currently 7 Research scholars are pursuing Ph.D. under his guidance in VTU. 